\title{
Cenário brasileiro das revistas eletrônicas de biotecnologia em acesso aberto com estratificação Qualis
}

\author{
Diego José Macêdo \\ Instituto Brasileiro de Informação em Ciência e Tecnologia \\ diegojmacedo@gmail.com \\ Ronnie Fagundes de Brito \\ Instituto Brasileiro de Informação em Ciência e Tecnologia \\ ronniefbrito@gmail.com \\ Milton Shintaku \\ Instituto Brasileiro de Informação em Ciência e Tecnologia \\ milton.shintaku@gmail.com \\ João de Melo Maricato \\ Universidade de Brasília \\ jmmaricato@gmail.com
}

\section{Como citar:}

MACEDO. D. J. et al. Cenário Brasileiro das revistas eletrônicas de Biotecnologia em acesso aberto com estratificação qualis. In: ABEC MEETING, 2, 2018, São Paulo. Anais... São Paulo: Associação Brasileira de Editores Científicos, 2018. p. 1-6.

http://dx.doi.org/10.21452/abecmeeting.2018.161

\section{RESUMO}

Os periódicos científicos, desde o seu nascimento, tem evoluído para disseminar informação, tornandose no canal preferencial para publicação de resultados de pesquisa de muitas disciplinas. Entretanto, pela quantidade de revistas disponíveis, tem-se a necessidade de verificar questões técnicas e de qualidade, que ajude a indicar aquelas mais significativas da área. Assim, o presente estudo visa obter o um quadro das revistas brasileiras de biotecnologia com Qualis, por meio de estudo com abordagem predominantemente quantitativo. A coleta de dados deu-se por meio da plataforma sucupira, cruzando dados com o DOAJ. Pode-se verificar a pouca representatividade das revistas brasileiras nos estratos mais alto do qualis e ratificar a interdisciplinaridade da biotecnologia. Mesmo preliminar, o estudo revelouse eficaz na sua metodologia, apresentando indícios que possibilitam a construção de um cenário básico das publicações com qualis em biotecnologia.

Palavras-chave: Qualis. Biotecnologia. Revistas brasileiras. Acesso aberto. 
"Cenário Brasileiro das revistas eletrônicas de Biotecnologia em acesso aberto com estratificação qualis"

\section{ABSTRACT}

The scientific journals, from its birth, have evolved to disseminate information, becoming without preferential channel for the publication of results of research of many disciplines. However, the amount of data available, the ability to read techniques and quality, is a most important indicator of quality in the area. Thus, the study aims to obtain a framework of Brazilian biotechnology journals with a predominantly quantitative approach. A data collection was done through the sucupira platform, crossing data with the DOAJ. A small representation of Brazilian journals can be verified in the higher strata of qualifying and ratifying an interdisciplinarity of biotechnology. The evaluation of the evaluation of this study in method has been determined in the methodological basis of publications with qualitis in biotechnology.

Keywords: Qualis. Biotechnology. Brazilian magazines. Open access.

\section{INTRODUÇÃO}

No Brasil, os periódicos científicos são relativamente recentes quando comparados com os primeiros europeus, nascidos ainda no século XVII. Como relata Freitas (2006), os primeiros periódicos científicos brasileiros datam do século XIX, com a chegada da tipografia no Brasil com a Imprensa Régia. Entretanto, pode-se afirmar que os periódicos científicos, com estrutura mais próxima aos atuais, tiveram início no século XX, mais precisamente na década de 1920.

A partir de então, os periódicos brasileiros tornaram-se porta voz do conhecimento científico produzido no país. Atualmente os periódicos brasileiros recebem destaque na América Latina, conforme consta no Latindex. Assim, quanto ao número de revistas científicas o país é destaque na região, mesmo que com certo isolamento linguístico, visto que grande parte das revistas latino americanas são publicadas em espanhol.

Para estratificar as revistas com critérios técnicos e de qualidade, a Coordenação de Aperfeiçoamento de Pessoal de Nível Superior (CAPES) criou, em 1996, o sistema Qualis. Este foi desenvolvido para avaliação de programas de pós-graduação, no qual os artigos são qualificados de forma indireta em razão qualidade das revistas em que são publicados. Mesmo com críticas por ser um índice de classificação feita à posteriori, apresenta um indicador técnico de qualidade, sendo um fator de seleção de periódicos pelos autores para a publicação dos resultados de suas pesquisas, assim como para busca por informações (BARATA, 2017; GARCIA; DUARTE, 2017; VIEIRA JUNIOR, 2017).

As revistas científicas onde os pesquisadores vinculados a programas de pós- graduação publicam seus trabalhos recebem a estratificação Qualis, que pode ser A1, A2, B1, B2, B3, B4, B5 e C. A CAPES classifica tanto revistas brasileiras como estrangeiras, variando em quantidade nas diferentes áreas do conhecimento. Em alguns casos, como por exemplo na física, a maioria é estrangeira. Em outras áreas do conhecimento reconhecidamente interdisciplinares, como é o caso da biotecnologia, pouco se conhece sobre as revistas, sobretudo as brasileiras de acesso aberto. Assim, o presente estudo tem por objetivo apresentar as revistas brasileiras de acesso aberto em biotecnologia estratificadas no sistema Qualis, de forma a compreender melhor a qualidade dessas revistas e as áreas do conhecimento com as quais a biotecnologia possui intersecção. 

qualis"

\section{METODOLOGIA}

O presente estudo tem aspectos descritivos, pois apresenta as características de um grupo de revistas científicas brasileiras de acesso aberto, que publicam temas relacionadas à biotecnologia. Para tanto, possui abordagem, majoritariamente, quantitativa, mesmo que analise alguns aspectos qualitativos.

A coleta de dados deu-se em duas etapas, sendo que a primeira foi: (1) extração dos dados das revistas da área de biotecnologia da Plataforma Sucupira; (2) extração dos dados de todas as revistas existentes no Directory of Open Access Journals (DOAJ). Por fim, cruzou-se essas duas bases, de forma estabelecer uma base consolidada de revistas brasileiras de acesso aberto da área de biotecnologia com classificação Qualis indexadas na base de dados DOAJ.

Por meio dessa base de dados consolidada pôde-se extrair as informações analisadas no presente estudo. Assim, mesmo que restrita às revistas estratificadas pelo Qualis e de acesso aberto, a amostra tende a ser representativa, na medida em que conta com as informações das revistas mais consolidadas, avaliadas pela CAPES.

\section{RESULTADOS}

Na plataforma Sucupira , mantida pela CAPES, a área de biotecnologia registra 4.376 revistas, entre nacionais e internacionais, em todos os estratos ( $A 1, A 2$, B1, B2, B3, B4, B5 e C). Entretanto, as informações disponíveis nesta plataforma são restritas, não permitindo verificar a sua origem, mas consta a informação do International Standard Serial Number (ISSN), que é um identificador da revista, a partir do qual dados complementares podem ser buscados em outras bases.

Por outro lado, a base DOAJ, por sua vez, registra informações de 11.551 revistas de acesso aberto cadastradas voluntariamente pelos editores de todo o mundo, sendo que 1.251 são revistas brasileiras, país que destaca-se pela sua representatividade, com mais de dez por cento de todos os registros na base DOAJ. Esse indício reforça a adesão brasileira no movimento de acesso aberto, sendo o expoente na América Latina em quantidade de revistas de acesso aberto.

Assim, ao se cruzar os dados dessas duas bases (Qualis e DOAJ), resultaram 548 revistas online e 386 revistas impressas em biotecnologia. Ao restringir para as revistas brasileiras, o número cai para 260 revistas online e 210 impressas, ou seja, pouco mais de $55 \%$ dos periódicos online e $44 \%$ dos periódicos impressos, mostrando uma ligeira predominância das revistas online em relação às revistas impressas na área de biotecnologia no Brasil.

Importa ressaltar que um dos critérios para a qualificação do Qualis é a quantidade de citação da revista, sendo diferenciado para revistas em suporte digital (online) e impressas. Assim, pode ser que a presença de revistas impressas nos estratos Qualis não seja confiável/fidedigno, pois é observado que as referências são, em sua maioria, de periódicos em suporte físico. Contudo, algumas revistas não possuem mais a sua versão em suporte físico, apenas suporte digital, mas ainda permanecem qualificadas com Qualis como impresso.

Da amostra de 470 revistas, levantou-se as informações sobre a estratificação Qualis e a área da revista, visto que a biotecnologia, como área de pesquisa, é interdisciplinar. Cabe citar que a Organização das Nações Unidas (ONU), em sua Convenção para a Biodiversidade, em 1992, definiu a Biotecnologia como sendo: 
"Cenário Brasileiro das revistas eletrônicas de Biotecnologia em acesso aberto com estratificação qualis"

"qualquer aplicação tecnológica que utilize sistemas biológicos, organismos vivos, ou seus derivados, para fabricar ou modificar produtos ou processos para utilização específica" (BRASIL, 1994).

Quanto à estratificação Qualis, destaca-se que nenhuma revista brasileira identificada na DOAJ é classificada nos estratos mais altos A1 e A2 (Quadro 1), revelando a predominância dos periódicos internacionais nesta área. No estrato $B 1$, apenas as revistas Memórias do Instituto Oswaldo Cruz do Instituto Oswaldo Cruz (Fiocruz) e Genetics and Molecular Biology da Sociedade Brasileira de Genética estão presentes, revelando a pouca presença de revistas brasileiras qualificadas de acesso aberto, na medida em que o Qualis apresenta 800 revistas B1 listadas.

Quadro 1 - Quantidade de revistas brasileiras de acesso aberto com Qualis em biotecnologia indexadas na base de dados DOAJ.

\begin{tabular}{ccccc|} 
Estrato Qualis & $\begin{array}{c}\text { Qtd revistas } \\
\text { brasileiras } \\
\text { Online }\end{array}$ & $\begin{array}{c}\text { Qtd revistas } \\
\text { brasileiras } \\
\text { Impressas }\end{array}$ & $\begin{array}{c}\text { Revistas com } \\
\text { qualis }\end{array}$ & $\begin{array}{c}\% \text { revistas } \\
\text { brasileiras no } \\
\text { estrato }\end{array}$ \\
\hline A1 & 0 & 0 & 428 & $0 \%$ \\
\hline A2 & 0 & 0 & 415 & $0 \%$ \\
\hline B1 & 2 & 3 & 800 & $0,62 \%$ \\
\hline B2 & 16 & 21 & 610 & $6,06 \%$ \\
\hline B3 & 40 & 58 & 477 & $20,54 \%$ \\
\hline B4 & 63 & 68 & 368 & $35,59 \%$ \\
\hline B5 & 80 & 38 & 479 & $24,63 \%$ \\
\hline C & 59 & 22 & 799 & $10,01 \%$ \\
\hline Total & 260 & 210 & 4376 & $10,74 \%$ \\
\hline
\end{tabular}

Fonte: Elaboração dos autores, 2018.

Nesse sentido, há indício de que a Biotecnologia comporta-se como as ciências rígidas, que dão preferência em publicar e citar periódicos estrangeiros. Em muitos casos ocorre pelo baixo fator de impacto dos periódicos nacionais, como crítica Rochae-Silva (2009), visto que periódicos nacionais têm dificuldades de sustentabilidade e, com isso, dificuldades em ser indexadas e serem pouco citadas por pesquisadores brasileiros.

$\mathrm{Na}$ base de dados DOAJ, no momento em que o editor realiza o cadastro da sua revista, há um campo para indicar os temas tratados pela revista, sendo que pode-se marcar mais que uma opção, muito utilizado para revistas interdisciplinares. Dessa forma, é possível classificar as revistas mantidas por essa base com esse metadado.

Nos dados coletados sobre as revistas brasileiras com qualis relativos a biotecnologia no DOAJ destacou-se a medicina como área de maior número de revistas, seguida por agricultura e ciência, que juntas totalizam mais de $50 \%$ das revistas. O Gráfico 1 apresenta a distribuição das revistas por área. Esses dados 
"Cenário Brasileiro das revistas eletrônicas de Biotecnologia em acesso aberto com estratificação qualis"

alinham-se as definições de biotecnologia apresentados por da Cunha e Melo (2009), destacando os aspectos relacionados à vida e a aplicação de técnicas.

Gráfico 1 - Distribuição das revistas por área do conhecimento.

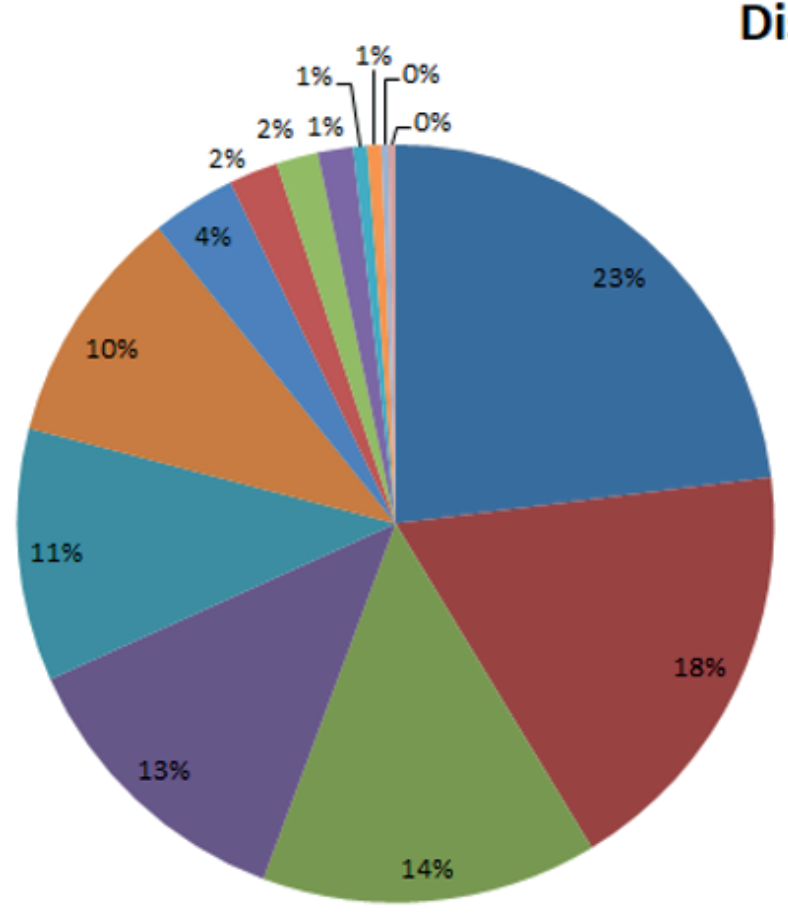

Distribuição das revistas por área.

Fonte: Elaboração dos autores, 2018.

Analisando somente as revistas online, notou-se que $46 \%$ das instituições possuem apenas uma revista com qualis em biotecnologia. Destaca-se apenas a Universidade de São Paulo (USP) com 14 revistas com qualis em biotecnologia, seguido pela Universidade de Goiás (UFG), com dez revistas e a Universidade Estadual Paulista Júlio de Mesquita Filho (Unesp) com nove revistas. Foi levantado que as 260 revistas são vinculadas a 158 instituições, sendo que 43 são de universidades, 24 de sociedades e 19 de associações, totalizando $55 \%$ das revistas. Cabe ressaltar que mesmo sendo o Qualis relacionado com os cursos de pós-graduação, parte das revistas classificadas não são ligadas a universidades.

Consolidando, tem-se que as revistas brasileiras classificadas com Qualis em biotecnologia, não ocupam os estratos de maior nível, assim como nas ciências rígidas, na qual grande maioria está vinculada às disciplinas aplicadas e pertencentes à universidades, associações e sociedades científicas. Assim, apresenta um cenário complexo, no qual não foi verificado a presença de periódicos próprios, o que pode representar pouco interesse científico, possuindo aspectos mais tecnológicos.

\section{CONSIDERAÇÕES FINAIS}

O presente estudo, mesmo que preliminar, apresentou um cenário desafiador para as revistas científicas brasileiras de acesso aberto, visto a baixa presença destas nos estratos mais altos do Qualis. O cenário também demonstra uma grande variedade de revistas, nas mais diversas áreas do conhecimento, que remete a um alto grau de interdisciplinaridade da área de biotecnologia, com destaque para as áreas aplicadas. 
Evidência as aplicações da biotecnologia pelas diversas áreas do conhecimento, apresentando indícios das áreas mais promissoras no desenvolvimento de técnicas e métodos para melhoria de processos ou produtos. Os periódicos classificados na biotecnologia, revelam ser instrumentos para desenvolvimentos em outras disciplinas, diante da variedade de estudos envolvendo biotecnologia publicados em revistas de diferentes disciplinas.

Por fim, diante das restrições do presente estudo, advoga-se por pesquisas mais aprofundados e abrangentes, voltadas aos periódicos brasileiros com classificação Qualis e de acesso aberto. Entretanto, como estudo preliminar revelou-se promissor ao estabelecer o cenário preliminar das revistas brasileiras classificadas com Qualis na área de biotecnologia e áreas do conhecimento de intersecção.

\section{REFERÊNCIAS}

BARATA, R. de C. B. Dez coisas que você deveria saber sobre o Qualis. RBPG, Brasília, v. 13, n. 30, p. 13 - 40, jan./abr. 2016 . Disponível em:<http://dx.doi. org/10.21713/2358-2332.2016.v13.947>. Acesso em: 12 jun. 2018

BRASIL. Congresso Nacional. Decreto Legislativo no 2, de 1994. Aprova o texto do Convenção sobre Diversidade Biológica, assinada durante a Conferência das Nações Unidas sobre Meio Ambiente e Desenvolvimento, realizada na Cidade do Rio de Janeiro, no período de 5 a 14 de junho de 1992. Coleção de Leis da República Federativa do Brasil, Brasília, DF, v. 3, p. 1354, 1994. Disponível em: <http://www2.camara.leg.br/legin/fed/decleg/1994/decretolegislativo-2-3-fevereiro-1994-358280-publicacaooriginal-1-pl.html>. Acesso em: 12 jun. 2018

CUNHA, Cleverson Renan da; MELO, Marlene Catarina de Oliveira Lopes. A confiança nos relacionamentos interorganizacionais: o campo da biotecnologia em análise. RAE electron., São Paulo, v. 5, n. 2, jul./dez. 2006. Disponível em: $<$ http://dx.doi. org/10.1590/S1676-56482006000200009 >. Acesso em: 12 jun. 2018

FREITAS, Maria Helena. Considerações acerca dos primeiros periódicos científicos brasileiros. Ci. Inf., Brasília, v. 35, n. 3, p. 54-66, set./dez. 2006. Disponível em: <https://doi.org/10.18225/ci.inf..v35i3.1113>. Acesso em: 12 jun. 2018

GARCIA, Leila Posenato; DUARTE, Elisete. Epidemiology and health services: journal of the Brazilian National Health System is classified B2 for public health at Qualis/Capes. Epidemiol. Serv. Saude, Brasília, v. 26, n. 2, p. 243-244, abr./jun 2017. Disponível em:<http://dx.doi.org/10.5123/s1679-49742017000200001>. Acesso em: 12 jun. 20182017

VIEIRA JUNIOR, Nilson Carlos. Boas práticas na construção de Periódicos Eletrônicos baseados no Sistema Eletrônico de Editoração de Revistas-SEER/ Open Journal Systems-OJS. [S.I.]: Biblioteca Latino-Americana (BIUNILA); Serviço de Gestão de Publicações Digitais-SGPD, 2017. Disponível em:<https://dspace.unila.edu.br/handle/123456789/3180>. Acesso em: 12 jun. 2018

ROCHA-E-SILVA, Mauricio. O novo Qualis, ou a tragédia anunciada. Clinics, São Paulo, v.64, n.1, p.1-4, 2009. Disponível em:<http://dx.doi.org/10.1590/S180759322009000100001>. Acesso em: 12 jun. 2018. 